Article

\title{
Smart NIR Tomoscopy to Predict Oxidative Stress in Rabbits
}

\author{
Alessia Candellone ${ }^{1}$, Pier Giorgio Peiretti ${ }^{2, *}$, Giorgio Masoero ${ }^{3}$ and Giorgia Meineri ${ }^{1}$ \\ ${ }^{1}$ Department of Veterinary Sciences, University of Turin, 10095 Grugliasco, Italy. E-Mails: \\ alessia.candellone@unito.it (A.C.); giorgia.meineri@unito.it (G.Me.) \\ ${ }^{2}$ Institute of Sciences of Food Production, National Research Council, 10095 Grugliasco, Italy. E-Mail: \\ piergiorgio.peiretti@ispa.cnr.it (P.G.P.) \\ ${ }^{3}$ Academy of Agriculture, 10100 Torino, Italy. E-Mail: giorgioxmasoero@gmail.com (G.Ma.) \\ * Correspondence: piergiorgio.peiretti@ispa.cnr.it; Tel.: ++39.11.6709233
}

Simple Summary: Near infrared spectroscopy (NIRS) fiber optics instruments have reached an efficient degree of portability, enabling their application in the study of animal products, both raw and transformed, food safety evaluation, and control. To date, however, little research has been carried out using NIRS as a tomoscopy instrument. To determine oxidative status in rabbits submitted to alimentary oxidative stress, a new smart-NIRS instrument was applied. The results showed that live ear-NIRS is a very time- and cost-effective method to assess and monitor oxidative status, before and during the experiment. This rapid and painless tool can be recommended for use in rabbit feeding protocols, where hard biological analyses are involved. Moreover, a practical extension of the SCÏO model in lactating and non-lactating animals showed an average spread of $33 \%$ of the oxidative risk in lactating does.

\begin{abstract}
The aim of this study was to find a correlation between in vivo NIR scan patterns, oxidative status, and blood serum metabolites in rabbits fed diets protected or unprotected against oxidation. Rabbits does in groups of eight were fed for 9 weeks with diets containing linseed, rich in polyunsaturated fatty acids (LS), or linseed plus hazelnut skins, with antioxidant function (LS+HS), and palm oil, rich in saturated fatty acids (PO). The animals were examined at days 1, 31, and 63 using a smart SCÏO molecular sensor, a new miniaturized web-based wireless device, applied to the internal ear flap (NIR range 740-1070 nm). The hazelnut peels integrated diet protected the rabbits from the oxidative stress induced by the addition of unprotected polyunsaturated fats. NIR tomoscopy was variously correlated with serum parameters, lysozyme $\left(\mathrm{R}^{2}=0.71\right)$, ROMs $(0.47)$, cholesterol (0.49), triglycerides (0.40), and a multivariate Index of Oxidative Risk (0.67 [IOR]). The correlations suggested a close connection between the clustering of the diets according to the laboratory variables and the NIRS scan pattern clustering at the ending trial day, as shown by the highly significant odds ratios. Advantageous use of this simple, painless technique was evident in the planning phase, with no difference among the groups at the beginning of the study, but an effect size that evolved differently over time until the end of the study. In a practical validation of the SCİO model in 92 commercial rabbit does, the average spread of the predicted IOR was $33 \%$ in lactating does $(2.54 \pm 0.05)$ vs. dry does $(1.91 \pm 0.07)$.
\end{abstract}

Keywords: rabbit; NIR tomoscopy; oxidative stress, blood serum parameters; error AKA confusion matrix.

\section{Introduction}

Health benefits in humans have been associated with the consumption of certain functional foods, particularly as regards animal products [1]. One of the easiest and most interesting ways to obtain functional properties in foods of animal origin is by modifying the animal's diet, which results in an increase of welfare for animals and a higher content of the desirable nutrients in their meat. 
Rabbit diet manipulation is very effective in increasing the level of fatty acids [2] and nutraceutical substances in body tissues [3]. Linseed, a potential commercial source of $\alpha$-linolenic acid, can be added in rabbit feed to obtain n-3 PUFA-enriched meat. Its addition has been shown to positively influence immune and cardiovascular function and to ameliorate regulation of blood pressure, cholesterol, and triglyceride levels in animals and humans [4].

While the effect of modifications to dietary feed on rabbit meat quality has been extensively studied, few reports describe the effects of diets on parameters related to animal welfare during breeding and monitoring of nutritional status and oxidative stress in vivo. Serum lipid profile and immune response can be evaluated as reliable indicators of animal welfare in vivo during rabbit farming. Furthermore, changes in oxidative status are also known to reflect physiological status [5-7] and correlate with an animal's ability to cope with dietary changes and stressful environmental stimuli. For these reasons, determination of oxidation and redox burden in live animals is gaining attention $[6,8]$. However, the main issue associated with in vivo screening of these markers, which require prolonged handling and restraint of farmed animals, is activation of the hypothalamicpituitary-adrenal axis, which leads to chronic stress with implications for production [9]. Furthermore, collection of blood samples can be time consuming, costly, and painful for the animal.

Given the above, live near-infrared reflectance spectroscopy (NIRS) applied to the inside aspect of the ear - as true NIR tomoscopy - could provide an effective, easy-to-use and non-invasive, painless solution to monitor animal health and welfare during farming. NIRS has been classically used in rabbit research to predict the nutritional parameters of feedstuffs [10,11] and the composition of hind-leg meat [12,13], to determine the intramuscular fat content in rabbit selection programs [14], and the composition of the fatty acids (FA) related to an organic production system [15]. A discriminating approach has been adopted in the experimental use of NIRS to ascertain technological or nutritional factors [16-21].

The effect of diet on the entire animal, as well as on the muscle tissue in rabbit, can be evaluated earlier from analysis of blood parameters related to the nutritional state and to the oxidative state [6]. In a previous companion paper [7], we evaluated the effects of linseed (LS) and hazelnut (Corylus avellana L.) skins supplementation (HS) on serum biochemical parameters [alanine aminotransferase (ALT), aspartate aminotransferase (AST), triglycerides and cholesterol], oxidative plasma status (as measured by the d-ROMs test and the OXI test), and immune function (lysozyme) in young rabbits, as in vivo parameters in relation to animal welfare. We found that redox imbalance was greatest in the rabbits fed the LS diet without additional antioxidant protection, with a significant increase in oxidative stress and decrease in antioxidant defenses. Furthermore, at the end of 9 weeks, lysozyme, an immune parameter linked to inflammatory phenomena, was higher in the group fed the LS diet than the groups fed the LS+HS or the palm oil (PO) diet. ALT remained unchanged in all three groups, while a significant increase in AST, triglycerides, and cholesterol was noted in the group fed the PO diet as compared with the other two groups. The aim of the present study was to find a correlation between in vivo NIR scan patterns, oxidative status, and blood serum metabolites in rabbits fed diets protected or unprotected against oxidation. Rabbit does in groups of eight were fed for 9 weeks diets containing LS, rich in polyunsaturated fatty acids, or LS+HS, with antioxidant function, or PO, rich in saturated fatty acids.

\section{Materials and Methods}

\subsection{Animals and Feeding}

The study was carried out as described in our previous study [7]. The rabbits were handled according to the principles stated in EC Directive 86/609/EEC regarding the protection of animals used for experimental and other scientific purposes. A total of 24 young grey Carmagnola rabbit does (6 months of age) with a mean body weight of $3901 \pm 9 \mathrm{~g}$ at the start (day 1) and of $3907 \pm 14 \mathrm{~g}$ at the end of the study period (day 63) were randomly assigned to one of three groups of eight rabbits per 
group. The rabbits were fed ad libitum with diets enriched with: linseed at $5 \%$ (LS) or LS at $5 \%$ and hazelnut peels at $1.5 \%(\mathrm{LS}+\mathrm{HS})$, or palm oil at $1.8 \%(\mathrm{PO})$, respectively.

The animals were examined at day 1 (T-0), day 31 (T-1), and day 63 (T-2) by means of a smart SCÏO molecular sensor (Consumer Physics Inc., Tel Aviv, Israel), a new miniaturized web-based wireless device to examine the internal ear flap (Figure 1) (NIR range 740-1070 nm); three spectra were replicated in each rabbit.

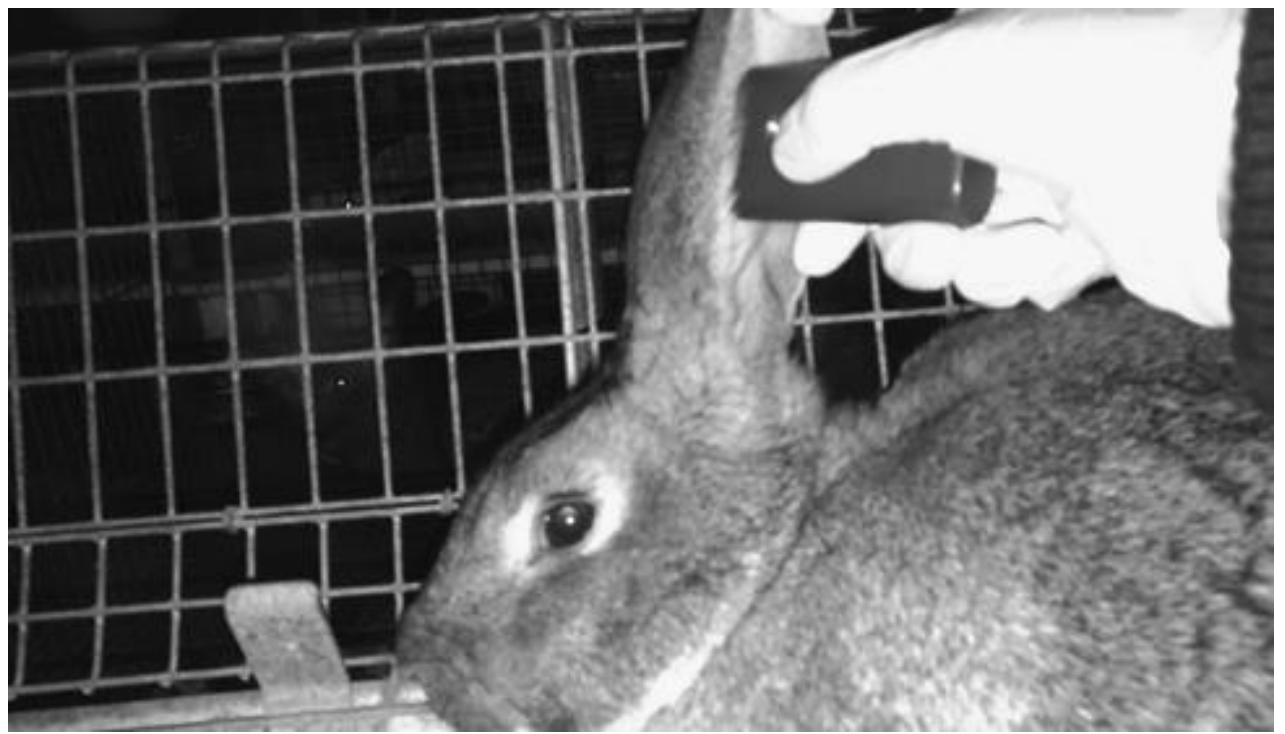

Figure 1. Rabbit examined with a pocket SCÏO molecular sensor.

\subsection{Biological Variables and a multivariate Index}

Eight biochemical variables (Table 1), which were described in detail in the previous paper [7], were measured. A synthetic Index of the Oxidative Risk (IOR) was computed from the eight serum variables for each animal. The index was formulated from a partial least square function using StatBox software (Grimmer Logiciels, Paris France), with two latent variables that fitted the numeric dummy values 1.0, for the PO and LS+HS diets, and 2.0 for the LS oxidized diet. The IOR values were then calibrated by the SCIIO software, yielding a PLS model that could predict from the NIR spectra the results of oxidative risk. This prediction was then applied to the spectra taken in the intermediate assessment at Time-1, when no blood sampling was performed.

\subsection{Statistics}

The chemometric analyses of the 331-point spectra were performed in two ways. First, qualitative discrimination of the three diets was realized using the proprietary software SCÏO-lab, based on AKA (Also Known As) confusion matrix, i.e., a reclassification similarity table of percentage occurrences, based on a random forest algorithm. Within the AKAs, the odds ratios (true positive/false negative) / (false positive/true negative) for each diet were computed using the online test provided by Medcalc software (Medcalc, Mariakerke, Belgium) to assess the probability of the NIRS in correctly discriminating for each single spectra the pertinent diet at each of the three time points. In order to signify the relationships among the three diets, for each time point, the AKAs matrices were clustered by the similarity cluster hierarchical analysis (CHA) method, as a Pearson correlation, using StatBox v. 6,5 software (Grimmer Logiciel, Paris, France).

A similar global representation of the three diets based on the laboratory variables was created by means of CHA of the standardized means of the three diets, calculated as Euclidean distance, by the software procedure.

Second, quantitative prediction of the analytes was performed on the spectra downloaded from the collection of the SCÏO repository; WinISI v. 1.04 software was then utilized, with standardization and first derivation math pretreatment. The Modified PLS calibration-cross-validation procedure was fitted to the laboratory variables, with filtering out of the outliers identified once when $t>2.0$. 


\subsection{Extension trial}

In corollary, a practical prediction of the SCIIO models was applied to the spectra obtained in a commercial rabbitry by rapid ear examination of 92 does, divided in two groups, according to whether lactating $(n=32)$ or not $(n=60)$.

\section{Results and discussion}

\subsection{Biological variables}

Table 1 presents the Pearson correlations matrix of the eight biological variables determined at days 1 and 63. Only four out of twenty-eight coefficients differed from zero at Time-0. Remarkably, the ROMs - OXI correlation was mild and positive (0.42), but at Time-2 it became highly negative (0.76). Similarly, the OXI - triglycerides and the ALT - lysozyme were 0.52 and -0.51 at Time- 0 , respectively, but both disappeared at Time-2. The key variable was the ROMs, which resulted strongly - negatively - concordant with the lysozyme level (0.85), but discordant - positively - with the saturated fatty indicators, i.e., cholesterol $(-0.52)$, triglycerides $(-0.59)$, and liver functionality (AST: -0.51). Even more discordant were these last parameters vs. the lysozyme level $(-0.73,-0.75$, and $-0.57)$. Obviously, the three lipid variables were highly inter-correlated (from +0.94 to +0.76 ), but, interestingly, the OXI were independent, the ROMs appeared slightly opposite (-0.51 to -0.56$)$, while the lysozyme level was in strong opposition to cholesterol (-0.73) and triglycerides $(-0.75)$.

Table 1. Pearson correlations matrix of the biological variables at day 1 (Time- 0 , below the diagonal) and at day 63 (Time-2, above the diagonal) with significant coefficients given in bold $(\mathrm{P}<0.05)$.

\begin{tabular}{cccccccccc}
\hline & ROMs & OXI & & Lysozym & ALT & Cholestero & Triglyceride & AST & IOR \\
Items & 1 & 2 & Ratio & $\mathbf{e}$ & $\mathbf{3}$ & $\mathbf{1}$ & $\mathbf{s}$ & 4 & $\mathbf{5}$ \\
\hline & & & & & & Time- 2 \\
\hline ROM & & & & & & & & & \\
s & 1 & $\mathbf{- 0 . 7 6}$ & $\mathbf{0 . 9 9}$ & $\mathbf{0 . 8 5}$ & -0.05 & $\mathbf{- 0 . 5 2}$ & $\mathbf{- 0 . 5 9}$ & $\mathbf{- 0 . 5 1}$ & $\mathbf{0 . 9 8}$ \\
OXI & $\mathbf{0 . 4 2}$ & 1 & $\mathbf{- 0 . 8 6}$ & $\mathbf{- 0 . 5 6}$ & 0.08 & 0.03 & 0.12 & 0.03 & $\mathbf{- 0 . 8 2}$ \\
Ratio & $\mathbf{0 . 6 7}$ & -0.40 & 1 & $\mathbf{0 . 8 4}$ & -0.06 & $\mathbf{- 0 . 4 4}$ & $\mathbf{- 0 . 5 1}$ & $\mathbf{- 0 . 4 2}$ & $\mathbf{0 . 9 9}$ \\
Lysoz & & & & & & & & & \\
. & -0.23 & -0.21 & -0.07 & 1 & -0.03 & $\mathbf{- 0 . 7 3}$ & $\mathbf{- 0 . 7 5}$ & $\mathbf{- 0 . 5 7}$ & $\mathbf{0 . 9 0}$ \\
ALT & -0.06 & 0.12 & -0.16 & $\mathbf{0 . 5 1}$ & 1 & -0.02 & 0.02 & 0.27 & -0.05 \\
Chol. & -0.15 & -0.02 & -0.13 & 0.12 & 0.34 & 1 & $\mathbf{0 . 9 4}$ & $\mathbf{0 . 7 6}$ & $\mathbf{- 0 . 5 4}$ \\
Trygl. & 0.08 & $\mathbf{0 . 5 2}$ & -0.33 & -0.35 & 0.18 & 0.34 & 1 & $\mathbf{0 . 7 7}$ & $\mathbf{- 0 . 6 1}$ \\
AST & 0 & 0.07 & -0.05 & 0.19 & -0.32 & 0.04 & -0.12 & 1 & $\mathbf{- 0 . 4 8}$ \\
\hline & & & Time- & & & & & & \\
\hline
\end{tabular}

${ }^{1} \mathrm{ROMs}=$ reactive oxygen metabolites. ${ }^{2} \mathrm{OXI}=$ serum antioxidants by the OXI test. ${ }^{3} \mathrm{ALT}=$ alanine aminotransferase. ${ }^{4} \mathrm{AST}=$ aspartate aminotransferase. ${ }^{5} \mathrm{IOR}=$ index of oxidative risk.

However, the patterns were clearly driven by the effects of diet. The last column in the Table 1 shows how highly relevant the oxidant/antioxidant indicators were for the IOR. The ALT level was not modified by time nor by diet, and it appeared independent of the other seven parameters at Time2 assessment.

Table 1 presents the patterns of the normalized means of the diets. The the diets had a considerable impact on the biological variables (Table 2). The LS diet boosted the ROMs to +5.4 units 
of standard deviation (SD) and the lysozyme level to +3.2 . The fatty acid saturated PO diet boosted the cholesterol and the triglycerides more than 4 units over baseline at T- 0 .

Table 2. Pattern of the normalized means of the diets for biological variables at day $63(\mathrm{~T}-2)$ and standardized partial least squares (PLS) coefficients.

\begin{tabular}{cccccc}
\hline Variables & $\begin{array}{c}\text { LS }^{1} \\
\mathbf{( 2 . 0}^{4}\end{array}$ & $\begin{array}{c}\mathbf{L S}^{4}+\mathbf{H S}^{2} \\
\mathbf{( 1 . 0 ) ^ { 4 }}\end{array}$ & $\begin{array}{c}\mathbf{P O}^{3} \\
\mathbf{( 1 . 0}^{4}\end{array}$ & $\begin{array}{c}\text { PLS } \\
\text { standardized } \\
\text { coefficients }\end{array}$ & x SD $^{\mathbf{5}}$ \\
\hline ROMs (CARR U) & $5.4^{\mathrm{b}}$ & $-2.7^{\mathrm{a}}$ & $-2.7^{\mathrm{a}}$ & 0.255 & 10 \\
OXI $(\mu$ mol HClO/l) & $-2.8^{\mathrm{a}}$ & $2.9^{\mathrm{b}}$ & $-0.1^{\mathrm{c}}$ & -0.262 & 4 \\
ROMs/OXI Ratio & 6.3 & -3.7 & -2.6 & 0.266 & 14 \\
ALT $(\mathrm{IU} / \mathrm{l})^{8}$ & 0 & 0 & 0 & 0.017 & 5 \\
Cholesterol $(\mathrm{mg} / \mathrm{dl})$ & $-2.5^{\mathrm{a}}$ & $-2^{\mathrm{a}}$ & $4.5^{\mathrm{b}}$ & -0.049 & 10 \\
Triglycerides $(\mathrm{mg} / \mathrm{dl})$ & $-2.6^{\mathrm{a}}$ & $-1.5^{\mathrm{a}}$ & $4.1^{\mathrm{b}}$ & -0.066 & 5 \\
AST $(\mathrm{IU} / \mathrm{l})^{9}$ & $-1.1^{\mathrm{a}}$ & $-0.8^{\mathrm{a}}$ & $1.9^{\mathrm{b}}$ & -0.028 & 5 \\
Lysozyme $(\mu \mathrm{g} / \mathrm{ml})$ & $3.2^{\mathrm{c}}$ & $-0.7^{\mathrm{b}}$ & $-2.5^{\mathrm{a}}$ & 0.195 & 5 \\
\hline
\end{tabular}

${ }^{1} \mathrm{LS}=$ linseed diet. ${ }^{2} \mathrm{LS}+\mathrm{HS}=$ linseed + hazelnut skins diet. ${ }^{3} \mathrm{PO}=$ palm oil diet. ${ }^{4}$ Dummy numerical coefficients assigned to the diets in the PLS model for the Index of Oxidative Risk; ${ }^{2}$ Multiple of the standard deviation (SD) at T-2 / T-0. ${ }^{6} \mathrm{ROMs}=$ reactive oxygen metabolites. ${ }^{7} \mathrm{OXI}=$ serum antioxidants by the OXI test. ${ }^{8} \mathrm{ALT}=$ alanine aminotransferase. ${ }^{9} \mathrm{AST}=$ aspartate aminotransferase.

As to the variability, time affected also the inner variance, which appeared steadily multiplied by 4 in OXI up to 14-fold in the ROMs/OXI ratio at T-2 (Table 2). The increase in variance was combined in parallel with that of the means; thus, the variation coefficient for the eight variables was, on average, $5 \pm 2 \%$ and $12 \pm 8 \%$ at T- 0 and T-2, respectively, but never appeared excessive as compared with published data [22-24].

As widely presented and discussed in our previous study [7], hazelnut peels integration was highly effective in protecting the rabbits from the oxidative stress induced by the high level of unprotected polyunsaturated fats: the oxidative stress was relatively low as compared with that measured in the rabbis fed the LS diet or the LS+HS diet, with a 35\% decrease in ROMs, a $19 \%$ increase in OXI, which means a $46 \%$ reduction in the ratio. Also, the lysozyme level was reduced by some $38 \%$. The ROMs were not modified in the rabbits fed the PO diet as compared with the ROMS measured in the rabbits fed the LS+HS diet, but the OXI was reduced by $8 \%$ and the ratio increased by $9 \%$. The lipidic footprint of the diets was markedly apparent, as the variations in the PO diet vs. the other two diets, not different, were contained $+125 \%$ in cholesterol, $+101 \%$ in triglycerides and $+56 \%$ in AST (Table 3 ).

Table 3. Discrimination ability of the eight biological variables and probability (threshold 33\%).

\begin{tabular}{ccccccccc}
\hline Items & ROMs $^{\mathbf{1}}$ & $\mathbf{O X I}^{\mathbf{2}}$ & Ratio & Lysozyme & ALT $^{\mathbf{3}}$ & Cholesterol & Triglycerides $^{\mathbf{A S T}^{\mathbf{4}}}$ \\
\hline Discrimination & & & & & & & & \\
$\%$ & 100 & 88 & 92 & 88 & 13 & 79 & 83 & 71 \\
$\mathrm{P}$ & $<0.0001$ & $<0.0001$ & $<0.0001$ & $<0.0001$ & 0.037 & $<0.0001$ & $<0.0001$ & 0.0001 \\
\hline
\end{tabular}

${ }^{1} \mathrm{ROMs}=$ reactive oxygen metabolites. ${ }^{2} \mathrm{OXI}=$ serum antioxidants by the OXI test. ${ }^{3} \mathrm{ALT}=$ alanine aminotransferase. ${ }^{4} \mathrm{AST}=$ aspartate aminotransferase.

When we excluded ALT, the linear discrimination of the diets based on each variable was very high, on average $86 \pm 9 \%$ with a maximum of $100 \%$ for the ROMs (Table 4 ). Note that ALT appeared as low-erratic with $13 \%$ vs. $33 \%$, showing a significant negative bias.

As synthesized by the cluster of the standardized means (Figure 2), the three diets were perfectly discriminated at day 63 (T-2), but with less distance between the LS+HS and the PO diet. This 
outcome can be clearly highlighted in the IOR values (Figure 3), where the values are highest for the rabbit does fed the LS diet.

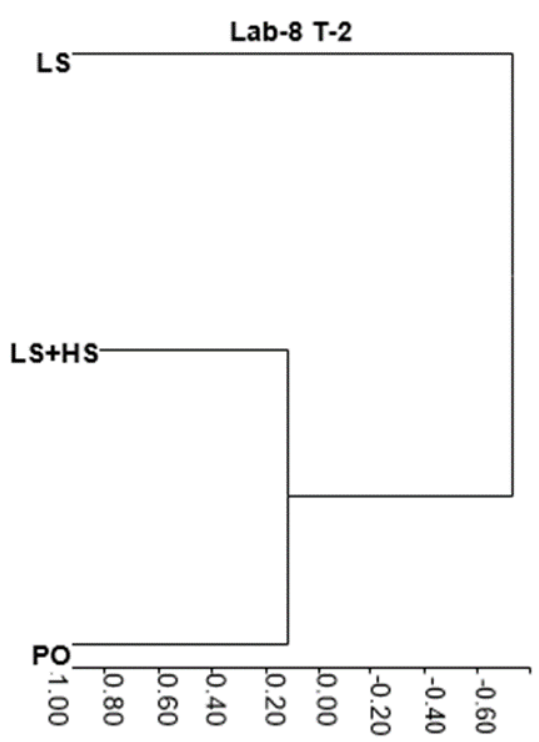

Figure 2. Similarity cluster of the three diets (LS = linseed diet; LS+HS = linseed+hazelnut skins diet; $\mathrm{PO}=$ palm oil diet) according to the eight means of the biological variables, at day 63 (T-2). Unit: Pearson correlation.

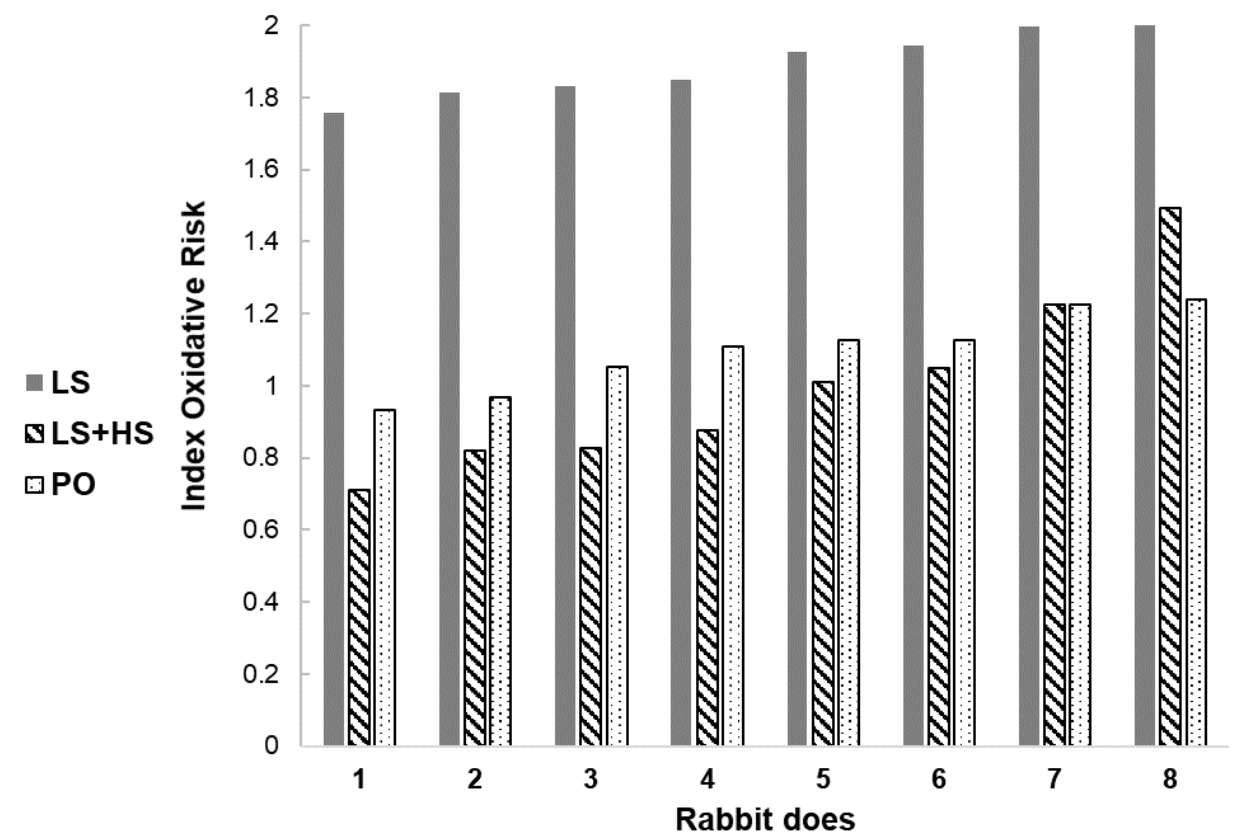

Figure 3. Histogram comparing the Index of Oxidative Risk in eight rabbit does fed one of the three diets (LS = linseed diet; LS+HS = linseed+hazelnut skins diet; PO = palm oil diet), measured at day 63 (T-2), ordered by ascendant values.

The risk of oxidative stress was demonstrated by Trebušak et al. [25]: after 52 days of unprotected dietary linseed oil administration at a very high level $(9 \% \mathrm{w} / \mathrm{w})$, the malondialdehyde (MDA) was increased by $38 \%$ in plasma, $132 \%$ in liver, and $210 \%$ in muscles. In a second experiment, with $6 \%$ dietary linseed oil, after 22 days of feeding Trebušak et al. [26] observed an MDA level in plasma that was half that in comparison with their previous trial and which was not changed with linseed oil diet, while, again, the MDA content was increased by $110 \%$ in liver and $257 \%$ in hindleg meat. In 
another study, Tres et al. [27] fed growing rabbits with 12 dietary treatments and observed that a LS supplement of $3 \%$ increased the lipid hydroperoxide values slightly in plasma $(+10 \%)$ but strongly in liver $(+151 \%)$ and meat $(+59 \%)$.

\subsection{NIR correlations}

Table 4 reports the performance of the NIR spectra collected at day 63 (T-2) in prediction and cross-validation of the biological variables. NIR tomoscopy was variously correlated with serum parameters, i.e., lysozyme ( $\mathrm{R}^{2}=0.71$; Figure 4$)$, $\mathrm{ROMs}(0.47)$, cholesterol $(0.49)$, triglycerides $(0.40)$, and IOR (0.67). The predictability of the biological variables from the NIR scan was statistically greater than zero for all the parameters but limited in laboratory utility. In 2008, a study in Harlan mice treated with streptozotocin showed that ventral NIR tomoscopy highlighted oxidative stress in normal and highly diabetic animals in good agreement $(\mathrm{r}=0.94)$ with laboratory analyses [20]. In live rabbits, dorsal NIR tomoscopy has been used to study physiological traits [28-30].

Table 4. Performance of the NIR spectra collected by NIR-SCÏO at day 63 (T-2) in predicting biological variables.

\begin{tabular}{|c|c|c|c|c|c|c|c|c|}
\hline Variables & Mean & $\mathrm{SD}^{1}$ & $\mathrm{CV} \%$ & $\mathrm{SEC}^{2}$ & $\mathrm{RSQ}^{3}$ & $\mathrm{SECV}^{4}$ & $\mathrm{RSQ}^{5}$ & $\mathrm{RPD}^{6}$ \\
\hline ROMs (CARR U) 7 & 343.8 & 71.7 & 21 & 49.51 & 0.52 & 52.06 & 0.47 & 1.4 \\
\hline OXI $(\mu \mathrm{mol} \mathrm{HClO} / 1)^{8}$ & 390.9 & 28.9 & 7 & 20.73 & 0.48 & 24.39 & 0.28 & 1.2 \\
\hline ROMs/OXI Ratio & 0.903 & 0.255 & 28 & 0.162 & 0.60 & 0.174 & 0.53 & 1.5 \\
\hline $\operatorname{ALT}(\mathrm{IU} / \mathrm{l})^{9}$ & 32.14 & 6.90 & 21 & 6.79 & 0.03 & 6.98 & 0.00 & 1.0 \\
\hline Cholesterol (mg/dl) & 41.01 & 16.46 & 40 & 9.34 & 0.68 & 11.67 & 0.49 & 1.4 \\
\hline Triglycerides (mg/dl) & 70.67 & 24.06 & 34 & 14.09 & 0.66 & 18.54 & 0.40 & 1.3 \\
\hline AST $(\mathrm{IU} / \mathrm{l})^{10}$ & 22.98 & 4.97 & 22 & 3.34 & 0.55 & 4.17 & 0.29 & 1.2 \\
\hline Lysozyme (g/ml) & 17.54 & 6.37 & 36 & 3.10 & 0.76 & 3.44 & 0.71 & 1.9 \\
\hline Index Oxidative Risk & 1.32 & 0.46 & 35 & 0.24 & 0.73 & 0.26 & 0.67 & 1.7 \\
\hline
\end{tabular}

${ }^{1} \mathrm{SD}=$ standard deviation. ${ }^{2} \mathrm{SEC}=$ standard error in calibration. ${ }^{3} \mathrm{RSQ}=$ root square coefficient in calibration. ${ }^{4} \mathrm{SECV}=$ standard error in cross-validation. ${ }^{5} \mathrm{RSQ} c \mathrm{v}=$ root square coefficient in crossvalidation. ${ }^{6} \mathrm{RPD}=$ ratio-performance deviation $(\mathrm{SD} / \mathrm{SECV}) .{ }^{7} \mathrm{ROMs}=$ reactive oxygen metabolites. ${ }^{8} \mathrm{OXI}=$ serum antioxidants by the OXI test. ${ }^{9} \mathrm{ALT}=$ alanine aminotransferase. ${ }^{10} \mathrm{AST}=$ aspartate aminotransferase.

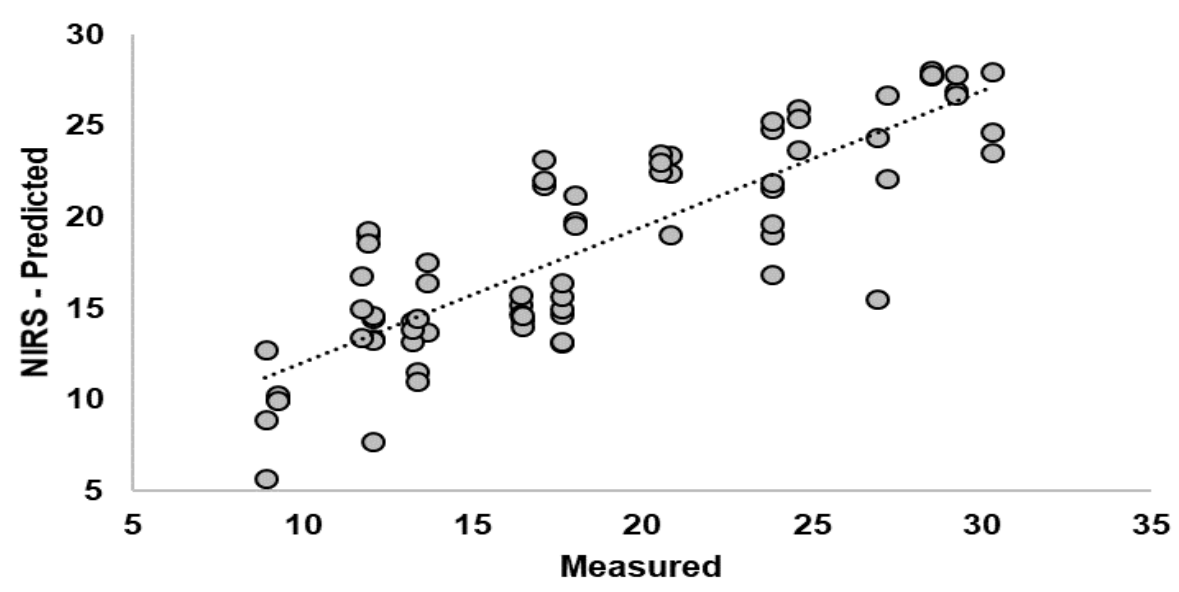

Figure 4. Performance of NIR tomoscopy in predicting lysozyme level $(\mu \mathrm{g} / \mathrm{ml})$ in serum at day 63 (T2). 


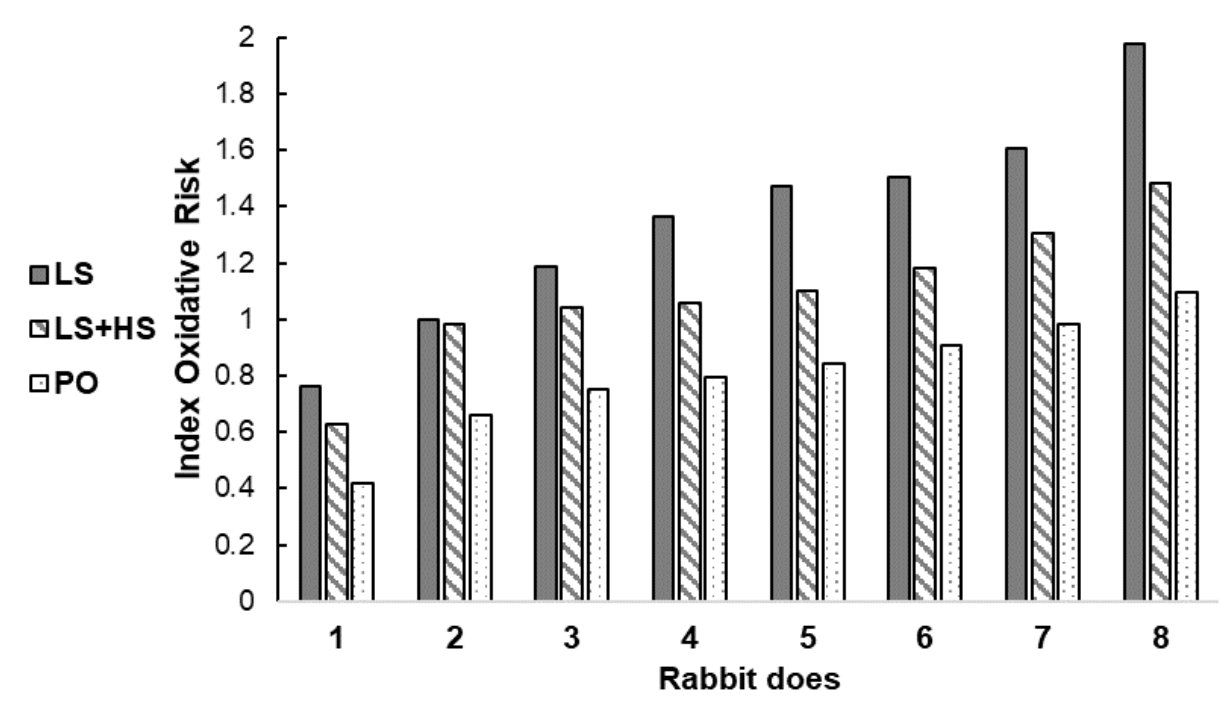

Figure 5. Histogram of the Index of Oxidative Risk, predicted in the eight rabbit does fed one of the three diets (LS = linseed diet; LS+HS = linseed+hazelnut skins diet; PO = palm oil diet), at day 31 (T1), ordered by ascendant values.

\subsection{AKA discrimination and clustering}

This study provides evidence for the profitability of live NIRS at the different steps of the experiment. When starting, after random allocation in groups, the rabbits assigned to receive the PO diet looked somewhat different from the others (Figure 6), but the odds ratio was not significant (Table 5), thus the groups were homogeneous, as confirmed by blood sampling and laboratory analyses.

At Time-1 (after 31 days) the pattern was changed, and the PO and the LS+Hs diets appeared to be significantly identified (P 0.004; Table 5) but not so the LS, which appeared near the LS+HS (Figure 6). At that intermediate step, no serum analyses were carried out. But we have the prediction for the IOR values from the NIR tomoscopy; and, in fact, Figure 5 shows that the does fed the PO diet had lower and homogeneous IOR values, while those fed the LS diet had the most inhomogeneous values, with a maximum range and mean, and those fed the LD+HS diet situated somewhere in between near those fed the LS diet.

Table 5. Confusion AKA matrix with percentages of classification and odds ratio of the three diets (LS = linseed diet; LS+HS = linseed+hazelnut skins diet; $\mathrm{PO}=$ palm oil diet), according to the NIRSCÏO spectra (N=72 spectra) at day 1 (T-0), day 31 (T-1), and day 63 (T-2).

\begin{tabular}{cccccccccc}
\hline Time & \multicolumn{3}{c}{ T-0 } & \multicolumn{3}{c}{ T-1 } & \multicolumn{3}{c}{ T-2 } \\
\cline { 2 - 10 } Diets & LS & LS+HS & PO & LS & LS+HS & PO & LS & LS+HS & PO \\
\hline PO & 38 & 47 & 27 & 19 & 8 & $\mathbf{6 2}$ & 22 & 35 & 27 \\
LS+HS & 22 & 25 & 39 & 42 & $\mathbf{6 0}$ & 16 & 26 & 31 & 43 \\
LS & 40 & 27 & 33 & 38 & 31 & 20 & $\mathbf{5 2}$ & 33 & 29 \\
\hline Odds $^{1}$ & 1.73 & 0.56 & 0.75 & 1.32 & 7.22 & $\mathbf{7 . 2 2}$ & $\mathbf{3 . 9 8}$ & 1.0 & 0.75 \\
P & 0.291 & 0.292 & 0.596 & 0.596 & 0.0004 & 0.0004 & 0.010 & 1.0 & 0.596 \\
\hline \multicolumn{8}{c}{ 'Odds = (true positive / false negative) / (false positive / true negative) }
\end{tabular}

But, 1 month later the situation changed and the diet LS appeared to differ the most from the other two, as shown from the T-2 NIRS cluster (Figure 6) and from the odds ratio 3.98 (P 0.01; Table 5). Importantly, the NIRS cluster at T-2 was very similar to the Lab-8 cluster in Figure 2, demonstrating a real and concrete connection between the live NIR ear-spectrum and the asset of the oxi-antioxidant as well as the lipid status. 


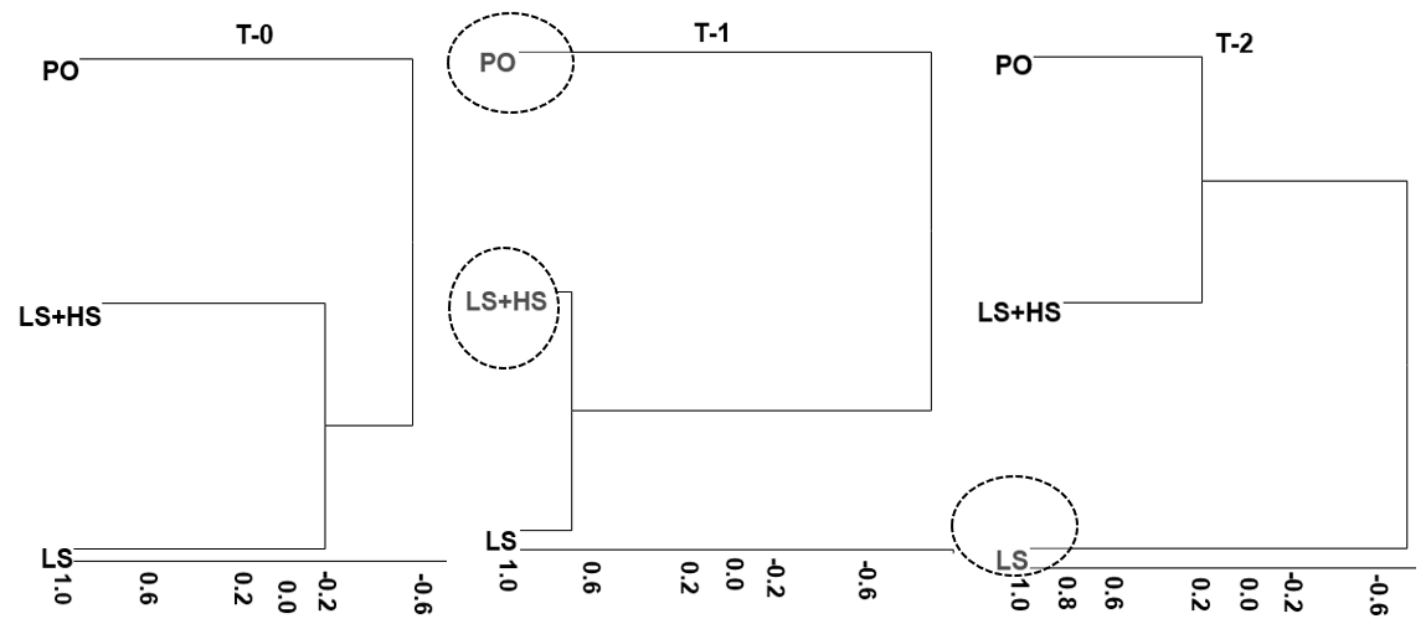

Figure 6. Similarity clusters of the three diets (LS = linseed diet; LS+HS = linseed+hazelnut skins diet; $\mathrm{PO}=$ palm oil diet) according to the confusion matrices from the NIR-SCÏO spectra at day 1 (T-0), day 31 (T-1), and day 63 (T-2). Unit: Pearson correlations.

3.4 NIR-SCÏO tomoscopy extension in a commercial sample of rabbit does.

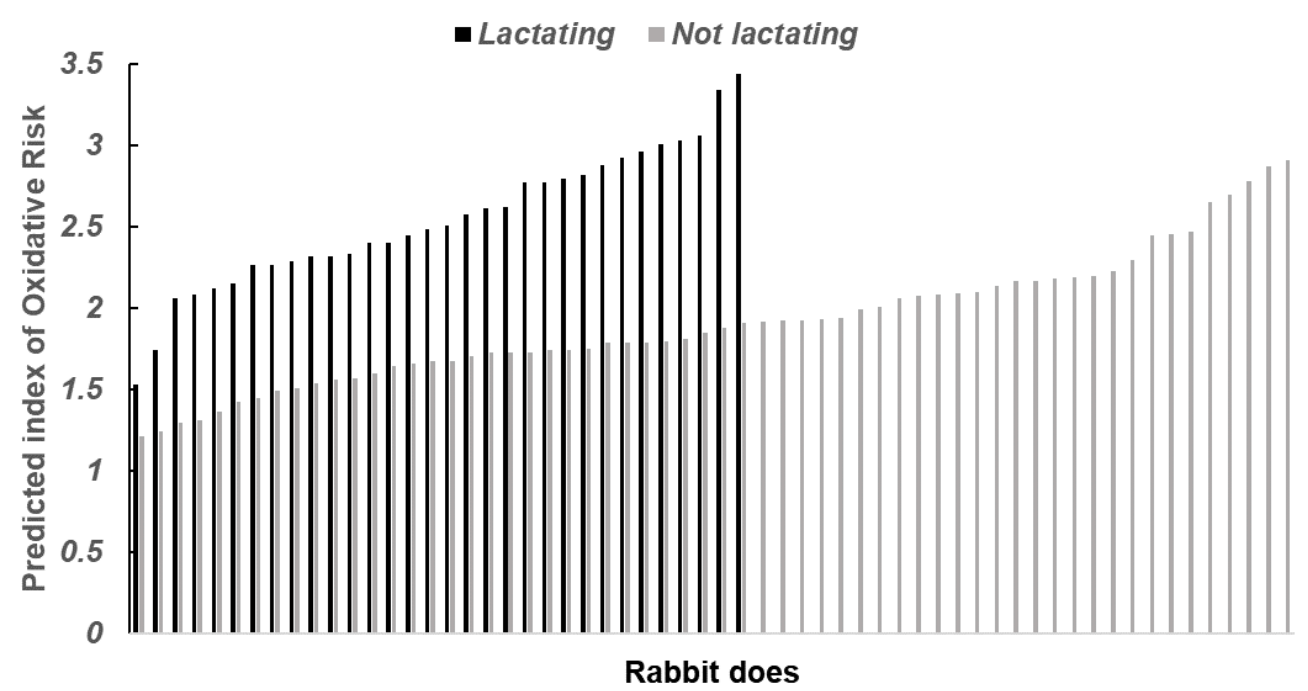

Figure 7. Extension of NIR-SCÏO tomoscopy in 92 rabbit does - lactating and not lactating - to predict the Index of Oxidative Risk. Histogram according to ascendant values.

In corollary, practical application of the SCÏO model in 92 commercial rabbit does (Figure 7) showed an average spread of $33 \%$ of the predicted IOR in lactating does $(2.54 \pm 0.05)$ vs. not lactating does $(1.91 \pm 0.07)$. A rare reference [22] reported that the lysozyme level increased by $66 \%$ in primiparous vs. nulliparous does. In caged, not particularly stressed growing hares, a phenylpropanoid glycoside-based natural extract of leaves of Verbenaceae diminished the ROMs by $>30 \%$ [31].

Oxidative stress is an active field of research in veterinary medicine and has been implicated in numerous disease processes, including sepsis, mastitis, acidosis, ketosis, enteritis, pneumonia, respiratory, and joint diseases [32]. After parturition, cholesterol has been signaled to increase by 5-6 fold [33]; in fact, extension of the experiment to the commercial herd showed predicted values of $23.3 \pm 1.9 \mathrm{mg} / \mathrm{dl}$ in the non-lactating and $25.3 \pm 2.9(+9 \%)$ in the lactating does (Figure 8). 


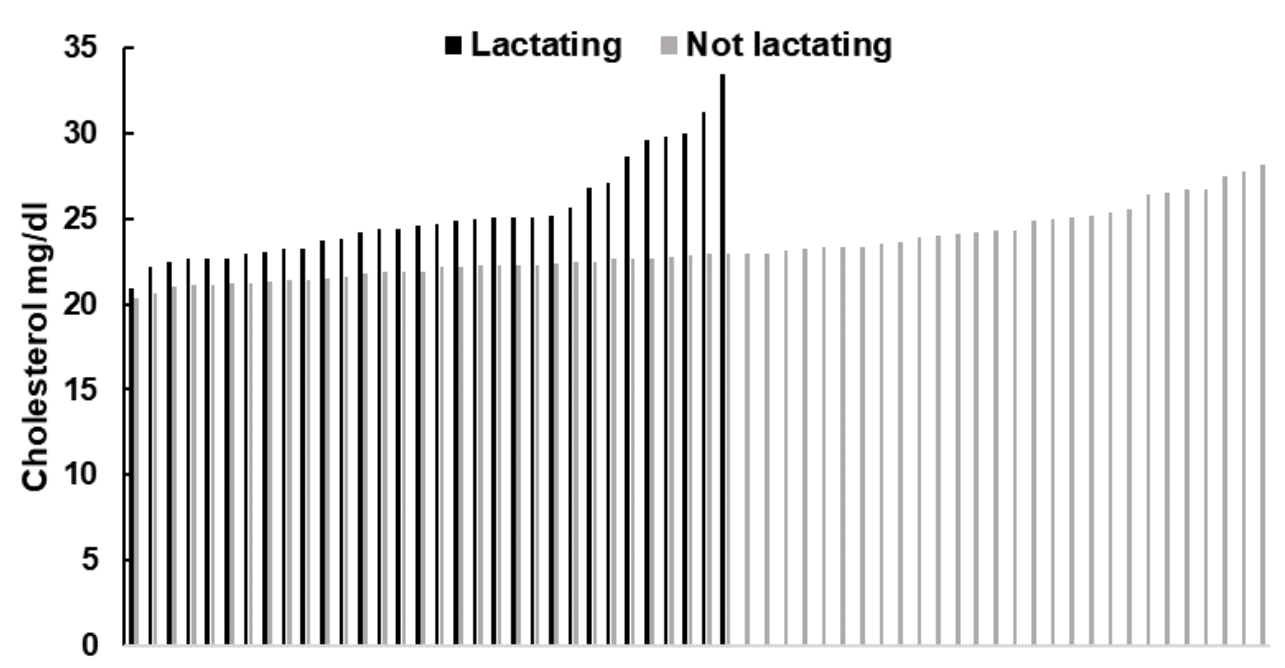

Rabbit does

Figure 8. Application of NIR-SCÏO tomoscopy in 92 rabbit does - lactating and not lactating - to predict the serum cholesterol. Histogram according to ascendant values.

\section{Conclusions}

Some years ago, Peiretti et al. [21] concluded a study on NIRS of body and tissues in growing rabbits fed diets with different fat sources and supplemented with Curcuma longa with the phrase "New portable NIRS devices could be profitably used to test discriminant dietary effects on rabbit meat". Availability of a totally new instrument, the NIR-SCÏO smart, cheap and complete, enabled us to reach the far shore. The device has been successfully proven able to discriminate mountain from plain dairy iced milk [34]. In the fight against commercial fraud, Wilson et al. [35] found SCÏO to be useful for identifying authentic artemisinin-based compounds and suggested that it could enable widespread drug screening at multiple points in the health network. In a recent review, Kademi et al. [36] discussed new NIRS devices that might stand as promising alternatives to large, expensive, benchtop types in food analysis, inspection, and control. NIR tomoscopy in live animals might eventually be a viable option

Author Contributions: A.C. was responsible for conducting the in vivo measurements, writing the manuscript. G.Me. ${ }^{1}$ was responsible for planning the study, conducting the in vivo measurements and chemical analysis, reviewing the manuscript. P.G.P. was responsible for planning the study, writing and editing the manuscript. G.Ma. ${ }^{3}$ was responsible for NIR validation, statistical analysis, writing and reviewing the manuscript.

Funding: This research was funded by the University of Torino (ex 60\%), grant number: UA.A200.D224 MEIG_RIC_LOC_15_01.

Acknowledgments: The author would like to thank Kenneth Britsch for language review of the manuscript.

Conflicts of Interest: The authors declare no conflict of interest.

\section{References}

1. Laparra, J.M.; Sanz, Y. Interactions of gut microbiota with functional food components and nutraceuticals. Pharmacol. Res. 2010, 61, 219-225.

2. Dalle Zotte, A. Perception of rabbit meat quality and major factor influencing the rabbit carcass and meat quality. Livest. Prod. Sci. 2002, 75, 11-32.

3. Dalle Zotte, A.; Szendro, Zs. The role of rabbit meat as functional food. Meat Sci. 2011, 88, 319-331.

4. Mozaffarian, D.; Wu; J.H. (n-3) fatty acids and cardiovascular health: are effects of EPA and DHA shared or complementary? J. Nutr. 2012, 142, 614S-625S.

5. Kaneko, J.J.; Harvey, J.W.; Bruss, M.L. Clinical biochemistry of domestic animals, 6th ed.; Academic Press: Amsterdam, The Netherlands, 2008. 
6. Meineri, G.; Giacobini, M.; Forneris, G. Evaluation of physiological parameters of the plasma oxidative status in rabbits. J. Appl. Anim. Res. 2017, 45, 315-319.

7. Candellone, A.; Peiretti, P.G.; Binello, A.; Bergagna, S.; Meineri, G. Effect of linseed diet and hazelnut skin supplementation on oxidative status and blood serum metabolites in rabbits. Prog. Nutr. 2019, 21, (in press).

8. Meineri, G.; Cornale, P.; Tassone, S.; Peiretti, P.G. Effects of Chia (Salvia hispanica L.) seed supplementation on rabbit meat quality, oxidative stability and sensory traits. Ital. J. Anim. Sci. 2010, 9, 45-49.

9. Tres, A.; Bou, R.; Codony, R.; Guardiola, F. Dietary n-6- or n-3-rich vegetable fats and $\alpha$-tocopheryl acetate: Effects on fatty acid composition and stability of rabbit plasma, liver and meat. Animal. 2009, 3, 1408-1419.

10. Xiccato, G.; Trocino, A.; De Boever, J.L.; Maertens, L.; Carabano, R.; Pascual, J.J.; Perez, J.M.; Gidenne, T.; Falcao-E-Cunha, L. Prediction of chemical composition, nutritive value and ingredient composition of European compound feeds for rabbits by near infrared reflectance spectroscopy (NIRS). Anim. Feed Sci. Technol. 2003, 104, 153-168.

11. Meineri, G.; Peiretti, P.G.; Masoero, G. Appraisal of ingestion and digestibility in growing rabbits using near infrared reflectance spectroscopy (NIRS) of feeds and faeces. Ital. J. Anim. Sci. 2009, 8, 75-82.

12. Pla, M.; Pascual, M.; Ariño, B. Protein, fat and moisture content of retail cuts of rabbit meat evaluated with the NIRS methodology. World Rabbit Sci. 2004, 12, 149-158.

13. Bázár, Gy.; Princz, Z.; Jekkel, G.; Locsmándi, L.; Andrássy-Baka, G.; Kövér, Gy.; Szendrő, Zs.; Romvári, R. NIRS prediction for protein and intramuscular fat content of rabbit hind leg meat. Agriculture. 2007, 13, 155-158.

14. Zomeño, C.; Hernández, P.; Blasco, A. Use of near infrared spectroscopy for intramuscular fat selection in rabbits. World Rabbit Sci. 2011. 19, 203-208.

15. Pla, M.; Hernández, P.; Ariño, B.; Ramírez, J.A.; Díaz, I. Prediction of fatty acid content in rabbit meat and discrimination between conventional and organic production systems by NIRS methodology. Food Chem. 2007, 100, 165-170.

16. Masoero, G.; Dal Bosco, A.; Castellini, C.; Sarti, L.; Bergoglio, G. Caractéristiques qualitatives et discrimination par NIR de muscles de lapins soumis à différents facteurs éxperimentaux. In Proc. Journées de la Recherche Cunicole, 19-20 November 2003, Paris, France, pp. 141-144.

17. Masoero, G.; Brugiapaglia, A.; Bergoglio, G.; Chicco, R. FT-NIR spectroscopy of fresh and treated muscle tissue in young female rabbits. In Proc. 8th World Rabbit Congress, 7-10 September 2004, Puebla, Mexico, pp. 1409-1415.

18. Masoero, G.; Sala, G.; Meineri, G.; Peiretti, P.G. NIRS discrimination of broiler rabbits fed with increasing levels of false flax (Camelina sativa L.) seeds in relationship to the fatty acid profiles. Ital. J. Anim. Sci. 2007, 6, 777 .

19. Masoero, G.; Sala, G.; Meineri, G.; Cornale, P.; Tassone, S.; Peiretti, P.G. NIR spectroscopy and electronic nose evaluation on live rabbits and on the meat of rabbits fed increasing levels of Chia (Salvia hispanica L.) seeds. J. Anim. Vet. Adv. 2008, 7, 1394-1399.

20. Masoero, G.; Sala, G.; Aragno, M.; Vercellinatto, I.; Danni, O.; Meineri, G. Impiego della spettroscopia NIR per una valutazione in vivo dello stress ossidativo in ratti STZ-diabetici: osservazioni preliminari. In Proc. $3^{\circ}$ Simposio italiano di Spettroscopia nel Vicino Infrarosso, Lazise, Italy, 22-23 May 2008, pp. 1-6.

21. Peiretti, P.G.; Meineri, G.; Masoero, G. NIRS of body and tissues in growing rabbits fed diets with different fat sources and supplemented with Curcuma longa. World Rabbit Sci. 2013, 21, 85-90.

22. Archetti, I.; Tittarelli, C.; Cerioli, M.; Brivio, R.; Grilli, G.; Lavazza, A. Serum chemistry and hematology values in commercial rabbits: preliminary data from industrial farms in northern Italy. In Proc. 9th World Rabbit Congress, Verona, Italy, 10-13 June 2008, pp. 1147-1151

23. Özkan, C.; Kaya, A.; Akgül, Y. Normal values of haematological and some biochemical parameters in serum and urine of New Zealand white rabbits. World Rabbit Sci. 2012, 20, 253-259.

24. Longato, E.; Meineri, G.; Peiretti, P.G. The effect of Amaranthus caudatus supplementation to diets containing linseed oil on oxidative status, blood serum metabolites, growth performance and meat quality characteristics in broilers. Anim. Sci. Pap. Rep. 2017, 35, 71-86.

25. Trebušak, T.; Levart, A.; Voljc, M.; Tomazin, U.; Pirman, T. The effect of linseed oil supplementation on performance, fatty acid composition and oxidative status of rabbits. Acta Agric. Slov. 2011, 98, 119-125.

26. Trebušak, T.; Levart, A.; Frankič, T.; Pirman, T. Effect of dietary linseed oil and Ganoderma lucidum or olive leaves supplementation on fatty acid composition and oxidative status of rabbits. World Rabbit Sci. 2014, 22, 71-81. 
27. Tres, A.; Nuchi, C.D.; Bou, R.; Codony, R.; Guardiola, F. Assessing rabbit and chicken tissue susceptibility to oxidation through the ferrous oxidation-xylenol orange method. Eur. J. Lipid Sci. Technol., 2009, 111, 563573.

28. Masoero, G.; Bergoglio, G.; Riccioni, L.; Destefanis, G.L.; Barge, M.T. Near Infrared Spectroscopy applied to living rabbits to estimate body composition and carcass and meat traits: a calibration study. J. Appl. Rabbit Res. 1992, 15, 810-818.

29. Masoero, G.; Bergoglio, G.; Giraudo, S.; Sala, G.; Barge, P.; Bardi, L.; Chicco R. Analisi ematiche innovative in allevamento ed in laboratorio: risultati preliminari in cinque allevamenti cunicoli. In Proc. I Convegno ASIC, Forlì, Italy, 30 September 2005, pp. 41-44.

30. Masoero, G.; Bergoglio, G.; Giraudo, S.; Sala, G.; Barge, P.; Bardi, L.; Chicco, R. Spettroscopia dell'area dorsale e del pelo del coniglio: risultati preliminari. In Proc. I Convegno ASIC, Forli, Italy, 30 September 2005, pp. 45-48.

31. Casamassima, D.; Palazzo, M.; Vizzarri, F.; Cinone, M.; Corino, C. Effect of dietary phenylpropanoid glycoside-based natural extracts on blood parameters and productive performance in intensively-reared young hares. Czech J. Anim. Sci. 2013, 58, 270-278.

32. Celi, P. Biomarkers of oxidative stress in ruminant medicine. Immunopharmacol. Immunotoxicol. 2011, 33, 233-240.

33. Minuti, A.; Bani, P.; Piccioli-Cappelli, F.; Uboldi. O.; Bacciu, N.; Trevisi, E. Metabolic and biochemical changes in plasma of the periparturient rabbit does with different litter size. Animal. 2015, 9, 614-621.

34. Battaglini, L.M.; Renna, M.; Lussiana, C.; Lombardi, G.; Probo, M.; Masoero, G. Smart near infrared spectroscopy on frozen milk samples can discriminate grass-fed from conventional milk. Ital. J. Anim. Sci. 2017, 16, 209-210.

35. Wilson, B.K.; Kaur, H.; Allan, E.L.; Lozama, A.; Bell, D. A new handheld device for the detection of falsified medicines: demonstration on falsified artemisinin-based therapies from the field. Am. J. Trop. Med. Hyg. 2017, 96, 1117-1123.

36. Kademi, H.I.; Ulusoy, B.H.; Hecer, C. Applications of miniaturized and portable near infrared spectroscopy (NIRS) for inspection and control of meat and meat products. Food Rev. Int. 2019, (in press). 